\title{
Nano-crystalline graphite for reliability improvement in MEM relay contacts ${ }^{\underline{k^{3}}}$
}

\author{
Sunil Rana ${ }^{1}$, Jamie D. Reynolds ${ }^{2}$, Ting Y. $\operatorname{Ling}^{2,3}$, Muhammed S. Shamsudin ${ }^{2,3}$, Suan Y. Pu ${ }^{2,3}$, Harold M. H. Chong ${ }^{2}$,
} Dinesh Pamunuwa ${ }^{1, *}$

\begin{abstract}
Micro- and Nano-electromechanical (MEM/NEM) relays can operate with zero-leakage at far higher temperatures and levels of radiation than transistors, but have poor reliability. This work demonstrates improvement in reliability of MEM relays using nano-crystalline graphite (NCG)-coated contact surfaces. The high stability of NCG in ambient air, along with its low surface energy, appear to make it an ideal contact material. NCG-coated relays achieved over 2.8 million fast, hot-switching cycles with a drain current of at least $5 \mu \mathrm{A}$ and on-resistance under $17 \mathrm{k} \Omega$, in ambient air. The relays also were tested in slow, hot-switching cycles designed to increase the electrical stress on the contact, and consistently achieved on-currents up to $50 \mu \mathrm{A}$ or the imposed current limit without failure. The eventual cause of failure appeared to be mechanical stress on the NCG layer over repeated cycling causing degradation. Increasing the layer thickness is expected to further improve the contact reliability. The relays are scalable and can be used as micro- or nano-scale switches in electronic components designed for very high temperatures and levels of radiation.
\end{abstract}

Keywords: microelectromechanial relay, nanoelectromechanial relay, nanocrystalline graphite, reliability, nanoelectromechanical relay contact, harsh environment electronics.

\section{Introduction}

Micro- and Nano-electromechanical (MEM/NEM) relays have zero leakage in the off-state as well as a much steeper subthreshold slope than transistors when turning 5 off [1]. Further, MEM/NEM relays can operate at a much higher temperature [2] and radiation levels (up to two orders of magnitude higher) [3] than transistors are capable of. Thus, MEM/NEM relays have great potential to be used as micro/nano switches to build logic circuits, 10 despite their relatively slow switching speed. For example, replacing pass transistors in interconnection switching blocks in FPGAs where the switching speed is irrelevant can achieve major energy savings 4. Alternately, circuits made entirely of relays can transform the capability of harsh-environment electronics where radiation hardness and high temperature operation take precedence.

The switching of a NEM relay requires the making and breaking of an electrically conductive physical contact between the source and drain terminals. The reliability of

\footnotetext{
Support from Innovate UK under grant 61931-453231, and the Malaysian Ministry of Higher Education for PhD scholarship is grate- ${ }_{40}$ fully acknowledged.

* Corresponding author

Email address: dinesh.pamunuwa@bristol.ac.uk (Dinesh Pamunuwa )

${ }^{1}$ Department of Electrical and Electronic Engineering, University of Bristol, Bristol BS8 1UB, UK

${ }^{2}$ School of Electronics and Computer Science, Univer- sity of ${ }_{45}$ Southampton, Southampton SO17 1BJ, UK

${ }^{3}$ University of Southampton Malaysia Campus, 79200, Iskandar Puteri, Johor, Malaysia
}

20 this contact poses one of the most significant challenges to the deployment of NEM relay technology. The contact (and hence the relay) tends to fail through a variety of mechanisms including increased resistance due to oxidation or wear of the conductive contact material, and microwelding or stiction leading to a permanently closed relay. A number of noble/transition metals (e.g. Pt, Ru, W) and metal oxides (e.g. $\mathrm{TiO}_{2}, \mathrm{RuO}_{2}$ ) have been tested to resolve the contact integrity issue [5, 6]; however, due to specific operational environment requirements (e.g. vacuum, dry nitrogen) or on-current limitations, none of these approaches seem to provide a robust contact.

The optimal contact material must:

- maintain mechanical/chemical integrity with sustained low 'on'-state resistance (ideally in an environment of ambient air);

- exhibit low surface energy to prevent stiction-related failure;

- have a well-characterized, repeatable synthesis process compatible with standard foundry offerings, and

- achieve a low-resistance, ohmic interface with the underlying material (if present) forming the conduction path.

Further, if deposited/grown in the form of a film, the contact material must also exhibit excellent adhesion to the substrate. Due to its inerntness in an ambient environment and low surface energy, different forms of carbon seem to satisfy many, if not all, of these requirements. 


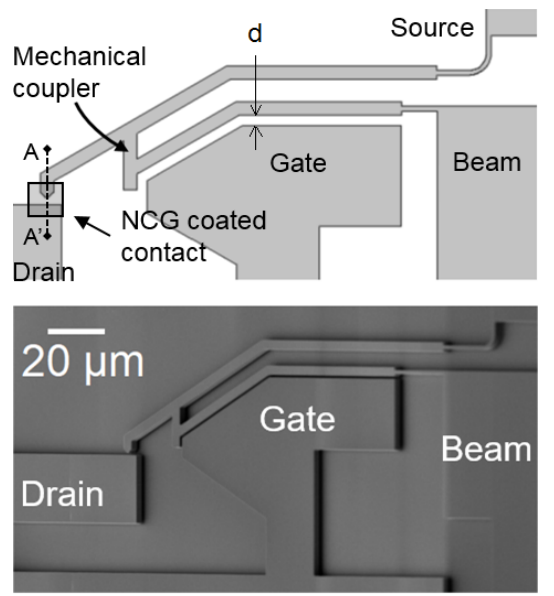

Figure 1: Relay architecture with a dual beam configuration (schematic and SEM micrograph). Current devices are 3-terminal devices where the source and beam terminals are connected via an electrically conductive mechanical coupler (i.e. etched out of the device silicon layer, same as the beams). Insertion of an insulating coupler will enable a single contact, 4 -terminal relay.

Grogg et al. reported amorphous-carbon-coated relays that benefit from the low surface energy of carbon [7, but require a relatively high initial voltage to be applied ${ }^{8}$ for filament formation, and dry $\mathrm{N}_{2}$ ambient. Loh et al. investigated a carbon nanotube cantilever and a stationary diamond-like carbon electrode [8], but the fabrication process does not appear to be easily adaptable for largescale fabrication. Thus, these approaches have progressed 90 the state-of-the-art in relay contact development; however, there are still unresolved issues related to repeatability, electrical conductivity and adhesion to substrate.

This work reports on the performance of a nano-crystalline form of carbon -nano-crystalline graphite (NCG)- that ${ }^{95}$ greatly improves the stability and reliability of MEM relays when used as a protective coating on the relay contacts. It is deposited using a highly optimized, repeatable plasma-enhanced chemical vapour deposition (PECVD) process, where layer thicknesses of $>200 \mathrm{~nm}$ can be read-100 ily achieved. The deposited NCG films have low surface roughness (5-8\% of the film thickness) and excellent adhesion to a variety of substrates, including silicon. High on-currents ( $>5 \mu \mathrm{A}$ were consistently achieved despite the relays being stored and cycled in an environment of am-105 bient air, showing the stability of NCG-coated relay contacts.

\section{Experimental Methods and Materials}

\subsection{Prototype Relay Architecture}

The relay architecture used for these experiments, shown in Fig. 1, top, consists of two parallel, cantilevered beams connected with a coupler. The free end of the beams makes an angle of $30^{\circ}$ with the horizontal while the fixed end has a narrow cross-section (region termed hinge). Similar to ${ }^{115}$
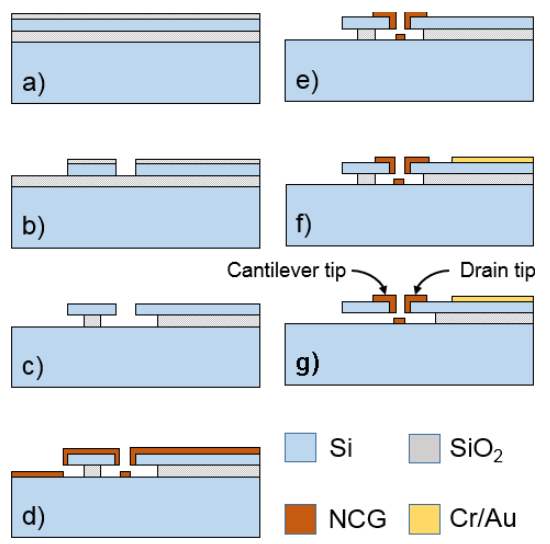

Figure 2: Fabrication process flow, across cross-section A-A', as shown in Fig. 1.top: a) SiO2 deposition for hard mask, b) hard mask and device silicon etch, c) partial etch of buried oxide layer, d) NCG deposition, e) NCG patterning, f) metal deposition for contact pads and g) final etch of buried oxide layer to release cantilever beams.

al. [10, the hinge acts as the stress concentration region. The stress, and hence the deformation, along the rest of the beam is minimal. The gate-to-beam gap, $d$, which is constant along the straight and angled length of the beam, is equal to the gap between the beam tip and the drain (the air gap along section AA' in Fig. 1-top). In the closed state of the relay, the angled beam architecture helps maintain a safe air gap between the beam and gate $(\sim 0.16 \times d$ for an angle of $\left.30^{\circ}\right)$ near the free end of the beam.

The dual-beam architecture can be used to realise a single-contact 4-terminal relay as long as the mechanical coupler is an insulator. In such a configuration, the signal voltage can be decoupled from the actuation voltage, enabling efficient digital circuit implementations compared to 3-terminal relays. The prototypes used in this study, however, which are aimed at evaluating NCG as an effective contact material, have a conducting silicon coupler.

In the fabricated prototypes (an example shown in Fig. 1 bottom), the horizontal projection of the beam length varies from 90 to $200 \mu \mathrm{m}$ and the corresponding beam stiffness varies from 1.41 to $0.2 \mathrm{~N} / \mathrm{m}$. The gate-to-beam actuation gap varies from 1 to $2 \mu \mathrm{m}$. The relatively large actuation gap, which is currently limited by our lithographic capabilities, necessitates a large footprint in order to reduce the actuation voltage. The actuation voltage for the fabricated designs varies between 15 and $45 \mathrm{~V}$. The architecture, however, is readily scalable where the overall footprint can be aggressively shrunk by reducing the actuation gap, with corresponding reductions in the key operational parameters of pull-in and pull-out voltages.

\subsection{Fabrication}

The fabrication process for the prototype relays is shown in Fig. 2, They were fabricated using silicon-on-insulator wafers with a silicon device layer thickness of $2 \mu \mathrm{m}$. The device silicon layer conductivity is $0.02 \Omega$-cm to $0.003 \Omega$ $\mathrm{cm}$. The buried-oxide layer is $1 \mu \mathrm{m}$ thick, while the handle 
silicon is $350 \mu \mathrm{m}$ with a conductivity of $1 \Omega$-cm to $10 \Omega$ cm. A $600 \mathrm{~nm}$ thick $\mathrm{SiO}_{2}$ hard mask was deposited using PECVD and patterned by a reactive ion etch (RIE). Subsequently, the device silicon layer was etched using an inductively-coupled plasma (ICP) etch step. The buriedoxide layer was then partially etched in buffered hydrofluoric (HF) acid (7:1). A $100 \mathrm{~nm}$ thick layer of NCG was subsequently deposited using PECVD.

The NCG was deposited by carrying out PECVD in $\mathrm{CH}_{4}$ and $\mathrm{H}_{2}$ plasma at $850{ }^{\circ} \mathrm{C}$ [1] and patterned using an oxygen-plasma RIE, such that only the beam tips and the adjacent drain regions were covered. Metal contact pads $(\mathrm{Cr} / \mathrm{Au})$ were then formed using sputtering and liftoff (for device testing). The partially suspended relays were finally fully suspended using buffered-HF-acid etch followed by critical-point drying.

\subsection{NCG: Friction Coefficient and Resistivity Measure- ${ }^{175}$ ment}

In order to study the tribological properties of NCGcoated surfaces a frictional force microscopy (FFM) system - Park XE7 AFM - was used. A contact-mode AFM $_{18}$ probe (PPP-CONTSCR, tip radius $<10 \mathrm{~nm}$ ) was used in dry-contact lateral-force mode, in ambient conditions. The friction coefficients were measured at four different normal force values 1, 2, 5 and $10 \mathrm{nN}$. At each normal force value, an average of three measurements was recorded. The friction coefficients for a $\mathrm{SiO}_{2}$-coated sample and a bare silicon sample were also measured for reference. The silicon sample was etched in buffered-HF acid immediately before the ${ }^{18}$ measurements to remove native oxide.

The thickness of the deposited NCG layers was measured using ellipsometry while the corresponding sheet resistance was measured using the four-point probe me-thod.

${ }_{150}$ Finally, the device on-resistance was analysed by applying ${ }^{190}$ a gate-source voltage greater than the pull-in voltage (i.e. pulling the beam tip into contact with the drain electrode), and measuring drain current $\left(I_{d}\right)$ with respect to varying drain voltage $\left(V_{d}\right)$. The drain voltage was ramped in steps of $8 \mathrm{mV}$.

\subsection{Hot-switch cycling experiments}

The prototype relays were first tested for their respective pull-in/out voltages $\left(V_{p i}, V_{p o}\right)$ by ramping up the gate voltage $\left(V_{g}\right)$ in steps of $200 \mathrm{mV}$ and monitoring the drain current $\left(I_{d}\right)$. Next, the conductance characteristics ${ }^{200}$ were established by applying a gate voltage $V_{g}>V_{p i}$ and sweeping the drain voltage $\left(V_{d}\right)$ across the range $-8 \mathrm{~V}$ to $8 \mathrm{~V}$ with the source grounded. Subsequently, two types of experiments were carried out: 1) high-resolution, slow $I_{d}-V_{g}$ sweeps and 2) low-resolution, fast cycling. The slow ${ }^{205}$ $I_{d}-V_{g}$ sweeps comprised ramping of gate voltage (in steps of $200 \mathrm{mV}$ ) within a range larger than the relay pull-in/out hysteresis window. The on-time during each cycle was $\sim 7$ s. In contrast, the low resolution fast cycling experi-
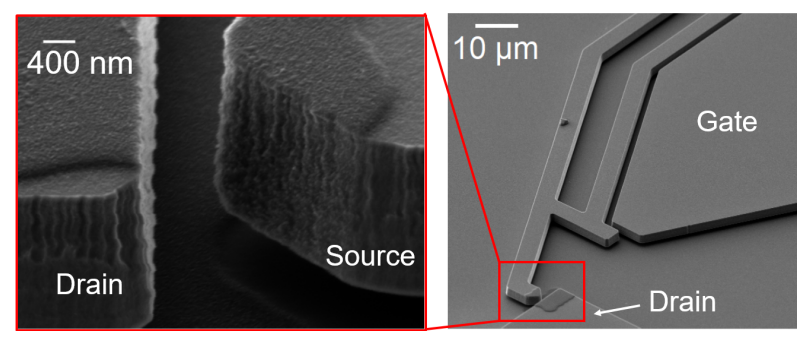

Figure 3: Relay source tip and drain coated with $100 \mathrm{~nm}$ thick NCG layer. The NCG layer has been lithographically patterned to cover the contact region only.

and a value just above the relay pull-in voltages. During the fast switching experiments, the relays were actuated at $90 \mathrm{~Hz}$ ( $5.5 \mathrm{~ms}$ wide pulses). Additional fast $I_{d}-V_{g}$ sweeps $(\sim 150 \mathrm{~ms}$ per sweep) were interspersed among the fast cycling experiments. These sweeps were used to monitor the change in the relay pull-in/out hysteresis window as the fast cycling progressed. An Agilent B1500A semiconductor parameter analyzer was used for the slow sweeps, while a dual channel Keithley 2602 source measure unit (SMU) was used for fast hot-switching. Both experiments were carried out in ambient air, at room temperature. The tested devices did not suffer mechanical failure during the cycling experiments.

\section{Results and Discussion}

A fabricated relay, with patterned NCG on the source tip and adjacent drain is shown in Fig. 3. Sidewall coverage is achieved, though the layer thickness does vary depending on the feature angle, as can be seen in Fig. 4. While the layer thickness is higher on flat regions and becomes thinner with increasing sidewall angles, the layer remains continuous. This is seen on the fabricated relays as well, where the NCG layer thickness is greater on the planar regions compared to the sidewalls (Fig. 3 inset).

\subsection{Characterisation of $N C G$}

The NCG characteristics were measured using Raman spectroscopy with excitation laser wavelength of $532 \mathrm{~nm}$. The Raman spectra (Fig. 5) show prominent D and G peaks which confirm the presence of $\mathrm{sp}^{2}$ hybridized carbon atoms. The presence of the broad second-order D peak, also known as the 2D peak, indicates a thick layer having a graphitic lattice structure. The presence of the shifted $2 D^{\prime}$ peak is due to the second-order intra-valley double resonance effect in the lattice structure, which confirms the presence of grain boundary defects and NCG [12. Fig. 6 shows a close-up image of a $40 \mathrm{~nm}$ layer of NCG deposited on a $\mathrm{SiO}_{2}$ substrate. The surface friction and electrical properties of deposited NCG layers on various substrates are discussed in Section 3.2 


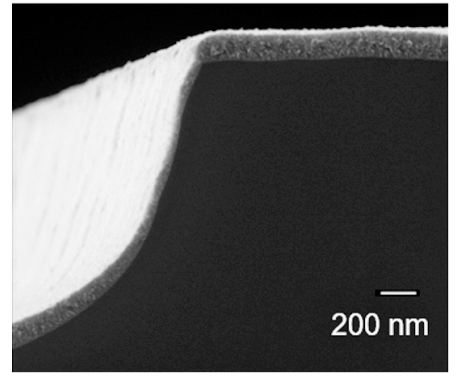

Figure 4: Helium ion microscopy image showing NCG thickness variation as a function of feature slope angle. The substrate is crystalline silicon. The layer thickness reduces for slopes close to 90 , but the layer remains continuous.

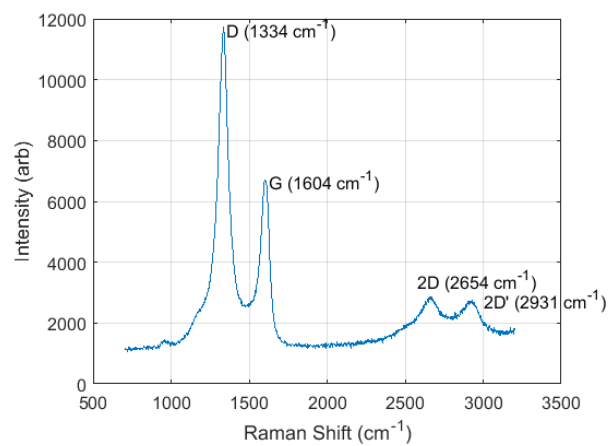

Figure 5: Raman spectra of NCG deposited on a Si substrate, over a $90 \mathrm{~nm}$ thick layer of $\mathrm{SiO}_{2}$ using PECVD at $850^{\circ} \mathrm{C}$.

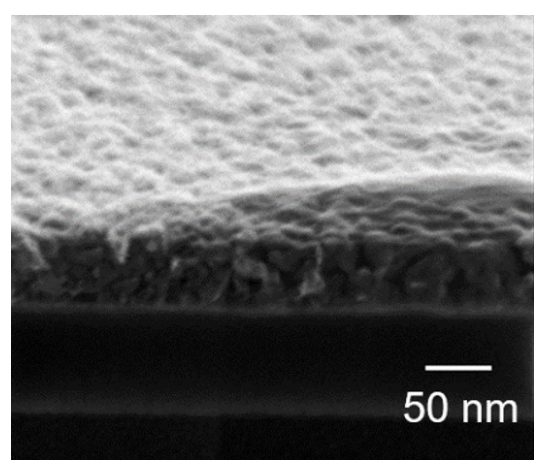

Figure 6: Helium ion microscopy image showing cross section of NCG deposited on a silicon dioxide substrate, where the crystalline structure is evident.

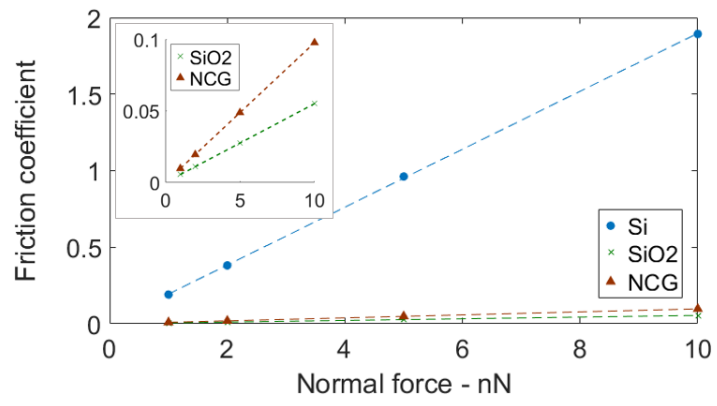

Figure 7: Friction force measurement for $\mathrm{Si}, \mathrm{SiO}_{2}$ on $\mathrm{Si}$ and NCG on $\mathrm{Si}$ substrates. The measured friction coefficient varies from 0.0055 for $\mathrm{SiO}_{2}$ to 0.1892 for $\mathrm{Si}$.

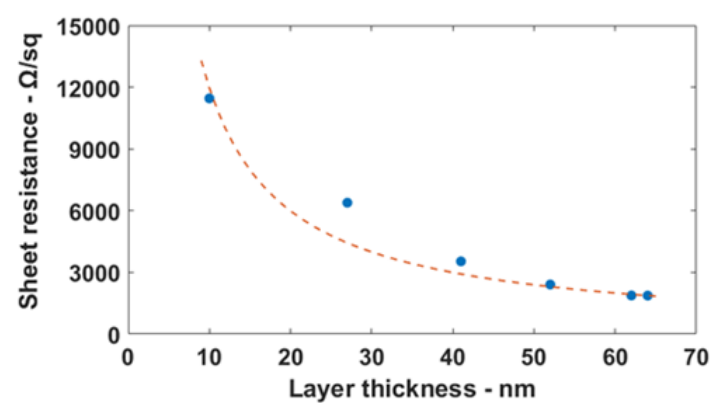

Figure 8: Variation in NCG sheet resistance with layer thickness. The dotted line represents a fit function inversely proportional to the layer thickness (negative power function).

\subsection{Friction coefficient and resistivity measurement}

A comparison of friction coefficients measured on $\mathrm{SiO}_{2}$, $\mathrm{Si}$ and NCG-coated Si substrates is shown in Fig. 7 and summarized in Table I. The $\mathrm{SiO}_{2}$ layer was thermally grown on clean $\mathrm{Si}$ samples in $\mathrm{O}_{2}$ atmosphere giving a layer thickness of $280 \mathrm{~nm}$. The NCG layer was deposited at $850^{\circ} \mathrm{C}$, in $\mathrm{CH}_{4}$ and $\mathrm{H}_{2}$ plasma for 60 min, giving a thickness of $47 \mathrm{~nm}$. The corresponding average surface roughness $\left(\mathrm{R}_{a}\right)$ of all three surfaces was also recorded.

While the average surface roughness of the freshly cleaned Si sample was low, it exhibited the highest friction coefficient. Despite the comparatively higher surface roughness the friction coefficient of the NCG layer was comparable to that of $\mathrm{SiO}_{2}$-roughly two orders of magnitude smaller than that of $\mathrm{Si}$.

The sheet resistance measurements for increasing NCG layer thickness are shown in Fig. 8. The sheet resistance roughly follows an inverse relationship with the layer thickness and values as low as $1870 \Omega / \square$ have been obtained for $\mathrm{a} \sim 64 \mathrm{~nm}$ thick layer.

\section{3. $I_{d}-V_{d}$ sweeps for conductance characterisation}

The drain current $\left(I_{d}\right)$ dependence on drain voltage $\left(V_{d}\right)$ is shown in Fig. 9. The relays consistently achieved on-currents greater than $50 \mu \mathrm{A}$, and for very high currents $(>0.5 \mathrm{~mA})$, the $I_{d}$ dependence on $V_{d}$ appears to be mildly non-linear. The on-resistance calculated over the range $-3 \mathrm{~V}$ to $3 \mathrm{~V}$ is $16.4 \mathrm{k} \Omega$. Other tested relays achieved 


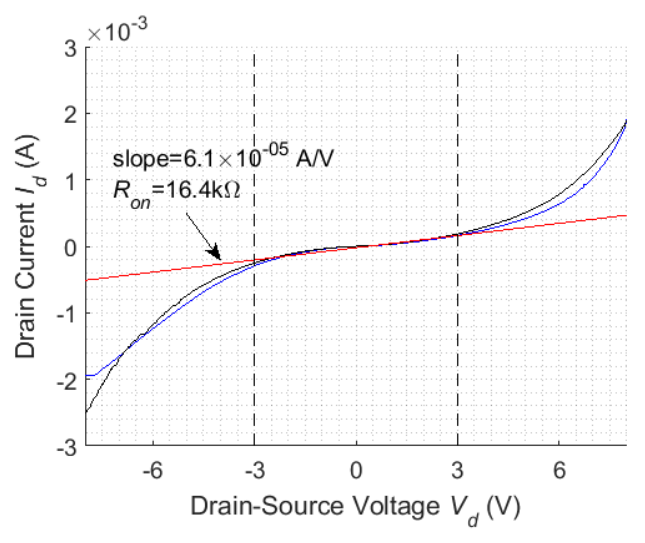

Figure 9: Slow $I_{d}-V_{d}$ sweep showing conductance characteristic for $40 \mathrm{~nm}$ thick layer. The relays were pulled-in $\left(V_{g}=23 \mathrm{~V}\right)$ before the $V_{d}$ sweep. The 'on' resistance in the range $-3 \mathrm{~V}$ to $3 \mathrm{~V}$ is $16.4 \mathrm{k} \Omega$. Over a wider range, the 'on' resistance is less. 'On' currents up to $50 \mu \mathrm{A}$ were consistently reached. Other tested devices showed similar, though not identical characteristics.

Table 1: A summary of the average surface roughness, and the corresponding friction coefficient, for bare, clean silicon and Si substrate coated with NCG

\begin{tabular}{lllll}
\hline Material & $\begin{array}{l}\text { Surface } \\
\text { ness } \\
(\mathrm{nm})\end{array}$ & $\begin{array}{r}\text { rough- } \\
\text { average }\end{array}$ & $\begin{array}{l}\text { Friction } \\
\text { cient }\end{array}$ & coeffi- \\
\hline Bare silicon & 0.1505 & & 0.1892 & \\
$\mathrm{SiO}_{2}$ & 0.051 & 0.0055 & \\
$\mathrm{NCG}$ on $\mathrm{Si}$ & 1.477 & & 0.00976 & \\
\hline
\end{tabular}

broadly similar, though not identical, conductance characteristics (residual stress causes the beam to bend slightly out of plane on release, resulting in the contact area being slightly different for different devices). Since there is $n^{275}$ rectifying behaviour, the relay conducts even at low drain voltages, a desirable characteristic in bulding efficient and low-power logic circuits. This is in contrast with amorphous carbon contacts demonstrated by Grogg et al. [7] which require carbon-filament formation to become con- ${ }^{280}$ ductive, at drain voltages of $1.5 \mathrm{~V}$ to $2.5 \mathrm{~V}$.

\subsection{High resolution, slow $I_{d}-V_{g}$ sweeps}

The slow $I_{d}-V_{g}$ experiments comprise 750 'long hotswitching cycles', where the relays remain in the 'on', con- ${ }^{285}$ ducting state, for $\sim 7 \mathrm{~s}$ during each cycle. The total on-time (i.e. drain conducting) is $\sim 5250 \mathrm{~s}$. The principal objective of this experiment is to place electrical stress on the relay. In the on-state, the source-drain contact region of the relay is expected to present the highest resistance to the ${ }^{290}$ source-drain current path. If present, Joule-heating related failure mechanisms (including microwelding), should manifest during these experiments. The slow sweep measurements are shown in Fig. 10. The current compliance limit is set at $1 \mu \mathrm{A}$, which is reached on every cycle. There is no appreciable change in the relay performance dur-

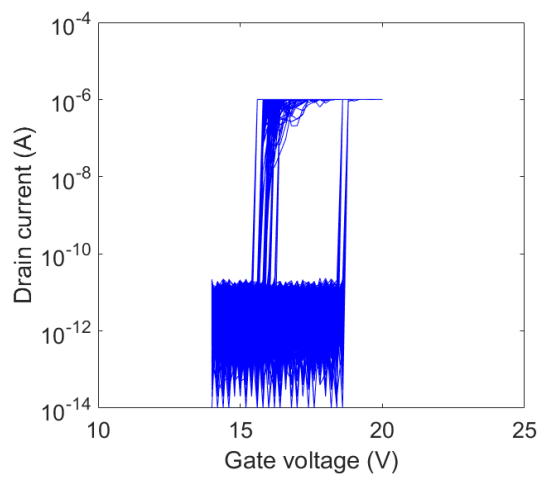

Figure 10: Slow $I_{d}-V_{g}$ sweep showing conduction for 750 cycles (relay 1). A drain current compliance limit of $1 \mu \mathrm{A}$ was imposed, which was hit every time. The periodic variation in the pull-in/out traces corresponds to the $200 \mathrm{mV}$ step size of the $V_{g}$ sweep. $V_{d}$ was kept at $1 \mathrm{~V}$.

ing this experiment, indicating that Joule-heating related degradation is unlikely to be a failure mechanism.

\subsection{Low resolution fast cycling with high resolution $I_{d}-V_{g}$ sweeps}

The low resolution fast cycling (or short hot-switching) experiment is carried out to place mechanical stress on the relays especially on the source-drain contact region. Most relays seemingly fail due to degradation of the contact surfaces. The fast cycling experiments should reveal failure mechanisms related to mechanical wear of the contact surfaces. Other contact failure mechanisms, such as surface oxidation or contamination, are considered exclusive of mechanical wear, which can also occur during storage.

The fast cycling measurements are shown in Fig. 11. where a bias of $1 \mathrm{~V}$ was applied across the drain and source, with a current compliance (i.e. limit) of $5 \mu \mathrm{A}$. The actuation signal comprises $5.5 \mathrm{~ms}$ wide pulses, giving a switching frequency of $90 \mathrm{~Hz}$. These measurements are low resolution where two $I_{d}$ measurements are recorded in the off-state and two in the on-state. The current compliance limit of $5 \mathrm{uA}$ is reached for over 2.8 million cycles with brief reductions in the on-current around 1.6 million and 2.05 million cycle marks. After 2.8 million cycles (an on-time of $>63650 \mathrm{~s}$ ) the relay continued to cycle with a reduced oncurrent, and did not suffer mechanical failure. Cycling was halted after $\sim 3.62$ million cycles. Other relays from the same fabrication batch that were tested achieved a similar number of fast hot-switch cycles, and a similar recovery phase was also observed after a small drop in on-current, for varying lengths of time.

Interspersed amongst the low resolution fast switching cycles are high-resolution $I_{d}-V_{g}$ sweeps where $V_{g}$ is swept in increments of $0.25 \mathrm{~V}$ and $I_{d}$ is recorded at every point. One such sweep is recorded after every 3600 fast cycles. These sweeps provide important insight into the change in the relay pull-in/out behaviour, with increasing switching cycles, which would otherwise not be observed with the 


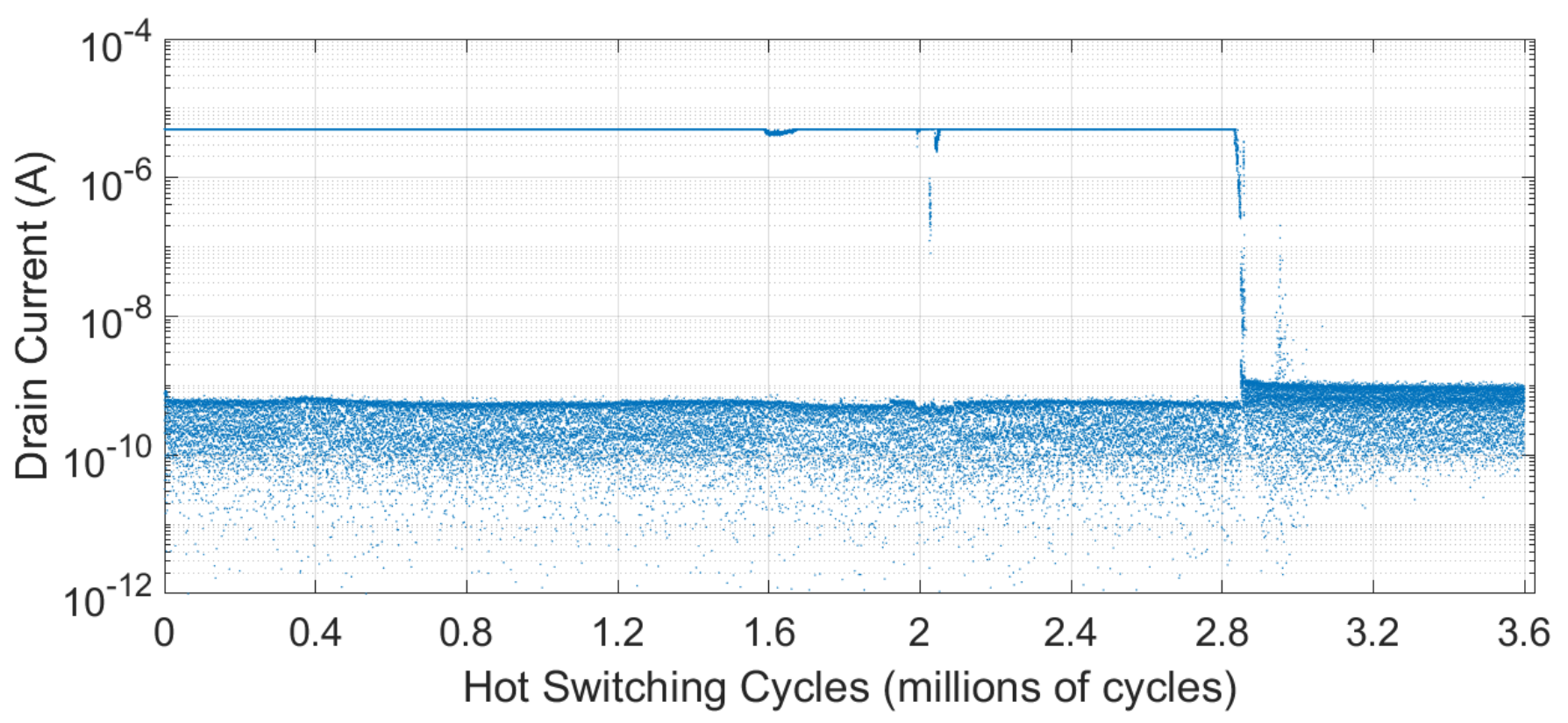

Figure 11: Low resolution, fast cycling results showing on-state and off-state drain current for each cycle. The drain voltage was kept at 1 V. and a drain current compliance limit of $5 \mu \mathrm{A}$ was set. The drain current limit was met for over 2.8 million cycles, with a brief blip at around 2.05 million cycles. After $\sim 2.8$ million cycles, the relay continued to cycle mechanically without failure, but did not meet the drain current compliance.

low-resolution, fast switching. The $I_{d}-V_{g}$ sweeps are collated in eight groups referred to as segments, (Fig. 12).

eight segments in total comprise a duration of $\sim 2.92$ million cycles $(\sim 66400 \mathrm{~s})$, and the segments are of equal time spans.

\subsection{Contact degradation}

335

As the experimental set-ups for the slow $I_{d}-V_{g}$ sweeps and the fast cycling experiments remained constant, it is assumed that any change in the on-current is due to contact degradation. The relay behaviour did not change appreciably during the slow $I_{d}-V_{g}$ sweeps, indicating 340 that Joule-heating related failure mechanisms may not be The on-current did change during the fast cycling experiments (Fig. 11), indicating that the sourcedrain contact evolves with repeated cycling. The momentary drop and recovery in drain current suggests a possible ${ }_{345}$ shift in the NCG layer at the contact. The crucial evidence comes from the $I_{d}-V_{g}$ sweeps conducted at regular intervals during fast cycling. During the first half of the experiment (Fig. 12a 12e the pull-out systematically decreases and the hysteresis window widens. This trend can 350 clearly be seen in Fig. 13 which plots the average pull-out, 320 i.e. the mean across all pull-out values in a segment, to avoid the effect of skewing by outliers.

The width of the pull-in/out hysteresis window is a function of device geometry and the surface forces acting between the contact surfaces [13. Since the geometrical parameters remain unchanged during the experiments, the contact surface forces seem to be responsible for the hysteresis window widening. Environmental contamination, particularly the formation of liquid thin films (leading to capillary forces), can increase surface forces; however, this explanation is discounted because such film formation can also happen during storage (the devices were stored in an ambient environment). Therefore, the most plausible explanation is the wear of the NCG layer at the contact surfaces leading to an increase in surface forces. This also explains the decrease in on-current during the fast cycling experiments.

Towards the end of segment $5(\sim 1.6$ million cycles $)$ the contact resistance starts to increase, as can be seen in Fig. 12e. The drain current then recovers, coinciding with the pull-out voltage increasing, suggesting a redistribution of NCG resulting in reduced surface forces. Following another short blip (Fig. 12f) and recovery phase (Fig. 12g), the pull-out reduces again (Fig. 12h), indicating degradation of the NCG layer. It appears that the impact stress over repeated cycling causes displacement of NCG, eventually thinning enough that the resistance increases significantly. The relays continued to cycle mechanically with near constant pull-in, until the experiments were halted. The experimental evidence suggests that NCG is an extremely effective anti-stiction coating. As can be seen in Fig. 3 the NCG layer thickness on the sidewalls is comparatively thin. Thus, it appears a thicker layer can be an effective solution to further improve the electrical integrity of the relay over its life-cycle.

\section{Conclusions}

The electrical and tribological properties of NCG were characterised to demonstrate that it is a suitable material for relay contact surface coatings. The friction coefficient 


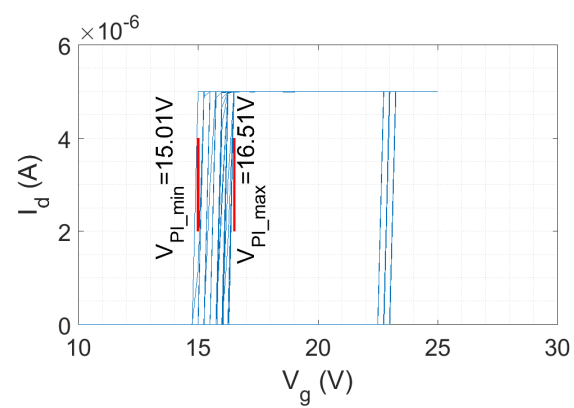

(a) Segment 1

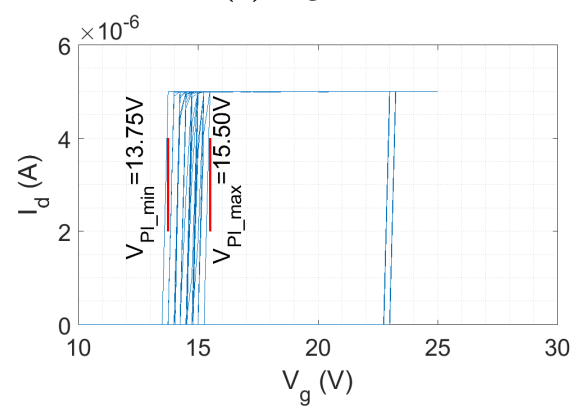

(d) Segment 4

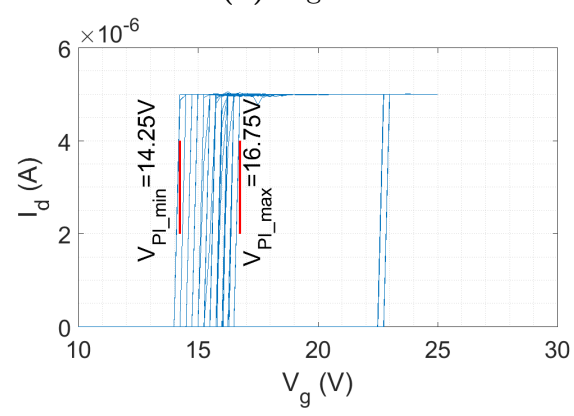

(g) Segment 7

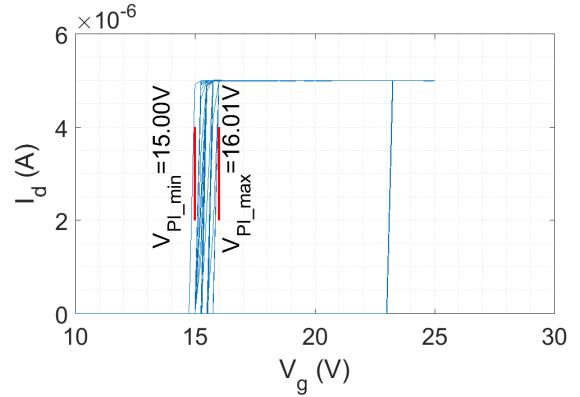

(b) Segment 2

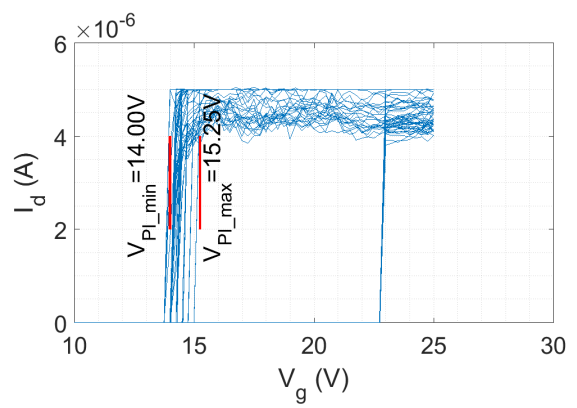

(e) Segment 5

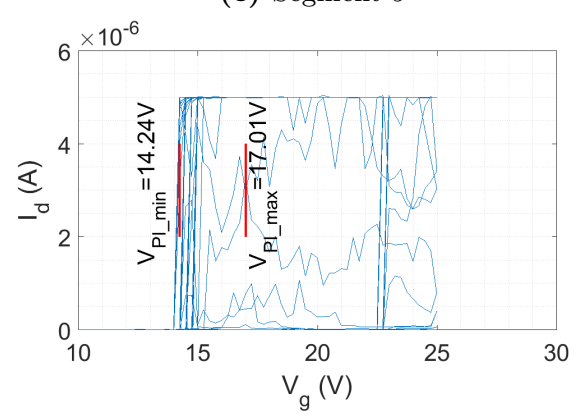

(h) Segment 8

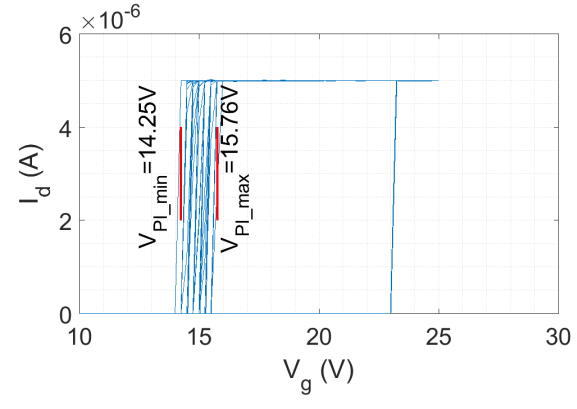

(c) Segment 3

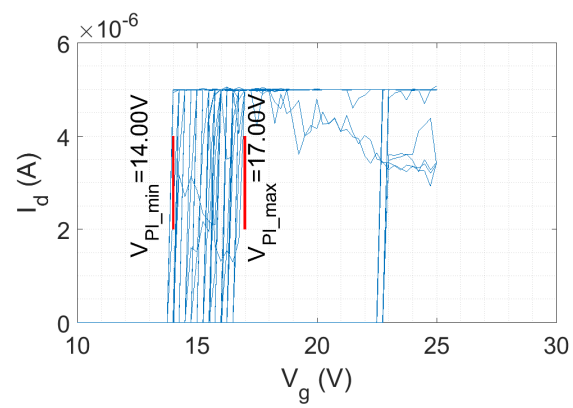

(f) Segment 6

Figure 12: Fast (high-resolution) sweep cycles were interspersed within the low-resolution fast cycles at regular intervals (after every 3600 cycles). The pull-out voltage consistently reduces until $\sim 1.6$ million cycles (e), when the drain current starts to drop. The widening of the hysteresis window over this period suggests an increase in surface-interaction forces at the source tip-drain interface. Then it recovers before undergoing a second current-reduction and -recovery phase ( $\mathrm{f}$ and $\mathrm{g}$ ). In segment $\mathrm{h}$, the electrical contact deteriorates again, coinciding with the pull-out reducing. For the noisy data in (h) the threshold for pull-in (out) was set as the drain current increasing (decreasing) beyond $2.5 \mu \mathrm{A}$, the mid-point of the compliance. The gate voltage was changed in steps of $0.25 \mathrm{~V}$, accounting for the deviation from the vertical seen on the graph. In reality, the slope is as near vertical as is possible to discern based on the limitations of the measuring equipment. The variation of the average pull-out voltage is shown in Fig. 13 


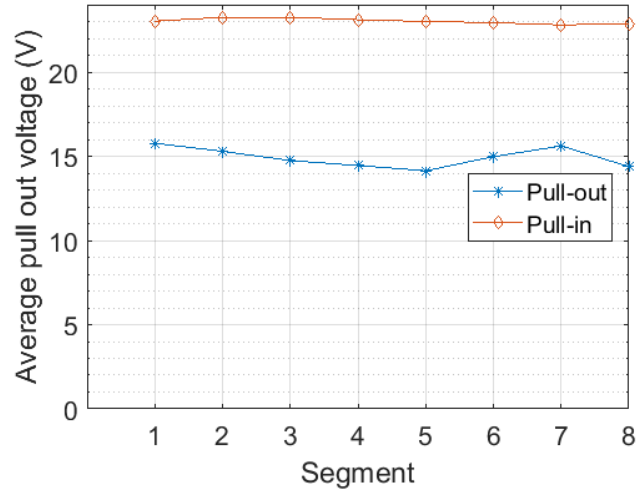

400

Figure 13: Variation of pull-out and pull-in voltage over eight equally-spaced segments over $\sim 3$ million cycles, averaged over each segment to reduce the effect of outliers. The pull-in remains virtually ${ }^{415}$ unchanged, while the pull-out steadily decreases indicating increases stiction, until the device recovers from a momentary blip. Once the recovery phase is over, the pull-out starts to decrease until failure.

of NCG layers is two orders of magnitude lower than that of silicon. Thus, it is an appealing anti-stiction coating material for relay contacts (as well as other MEMS applications). A layer of NCG deposited directly on highly doped n-type silicon (resistivity: $0.02 \Omega$-cm to $0.003 \Omega$-cm) appears to readily form an ohmic junction. This signifiantly simplifies the relay fabrication process by obviating the need for conductive metal or silicide layers. Thus, the residual stresses introduced by metal/silicide layers can be completely avoided. The NCG deposition process is well optimized, enabling precise control over layer thicknesses. Crucially, the relays were stored and tested in ambient air, indicating that NCG coated contacts were inert and stable in an ambient environment. Thus, NCG-coated relays may reduce the need for complex and expensive hermetic ${ }_{440}$ packaging solutions.

NCG-coated relays presented here demonstrated a stable electrical contact and maintained a current of $5 \mu \mathrm{A}$ in sustained switching for over 2.8 million cycles. A de- ${ }_{445}$ tailed qualitative study of the NCG coated contact, however, indicates that repeated cycling gradually affects the n-resistance as well as the surface force. This is most likely due to mechanical degradation of the NCG layer ${ }_{450}$ on the contact surface. The characterisation of the deposited NCG layer indicates that a thicker sidewall layer should provide further improvements in contact performance and reliability. The work presented here demon-455 strate how monocrystalline silicon relays, with highly desirable mechanical properties, can be coated with NCG to realise reliable contacts, the main point of failure that has held back their deployment.
[3] H. R. Shea, Effects of radiation on MEMS, in: Proc. SPIE MOEMS-MEMS, 2011, pp. 79280E-79280E.

[4] C. Chen, R. Parsa, N. Patil, S. Chong, K. Akarvardar, J. Provine, D. Lewis, J. Watt, R. T. Howe, H.-S. P. Wong, S. Mitra, Efficient fpgas using nanoelectromechanical relays, in: Proceedings of the 18th Annual ACM/SIGDA International Symposium on Field Programmable Gate Arrays, FPGA '10, ACM, New York, NY, USA, 2010, pp. 273-282. doi: 10.1145/1723112.1723158 URL http://doi.acm.org/10.1145/1723112.1723158

[5] I. R. Chen, Y. Chen, L. Hutin, V. Pott, R. Nathanael, T. J. K. Liu, Stable ruthenium-contact relay technology for low-power logic, in: 2013 Transducers Eurosensors XXVII: The 17th International Conference on Solid-State Sensors, Actuators and Microsystems (TRANSDUCERS EUROSENSORS XXVII), 2013, pp. 896-899. doi:10.1109/Transducers.2013.6626912

[6] M. P. de Boer, D. A. Czaplewski, M. S. Baker, S. L. Wolfley, J. A. Ohlhausen, Design, fabrication, performance and reliability of pt- and ruo 2 -coated microrelays tested in ultra-high purity gas environments, Journal of Micromechanics and Microengineering 22 (10) (2012) 105027.

URL http://stacks . iop. org/0960-1317/22/i=10/a=105027

[7] D. Grogg, C. L. Ayala, U. Drechsler, A. Sebastian, W. W. Koelmans, S. J. Bleiker, M. Fernandez-Bolanos, C. Hagleitner, M. Despont, U. T. Duerig, Amorphous carbon active contact layer for reliable nanoelectromechanical switches, in: Proc. Int. Conf. Micro Electro Mechanical Systems (MEMS), 2014, pp. $143-146$.

[8] O. Loh, X. Wei, C. Ke, J. Sullivan, H. D. Espinosa, Robust carbon-nanotube-based nano-electromechanical devices: Understanding and eliminating prevalent failure modes using alternative electrode materials, Small 7 (1) (2011) 79-86. doi: $10.1002 / \mathrm{smll} .201001166$ URL http://dx.doi .org/10.1002/smll.201001166

[9] R. Parsa, M. Shavezipur, W. S. Lee, S. Chong, D. Lee, H. S. P. Wong, R. Maboudian, R. T. Howe, Nanoelectromechanical relays with decoupled electrode and suspension, in: 2011 IEEE 24th International Conference on Micro Electro Mechanical Systems, 2011, pp. 1361-1364. doi:10.1109/MEMSYS.2011. 5734687

[10] D. Grogg, U. Drechsler, A. Knoll, U. Duerig, Y. Pu, C. Hagleitner, M. Despont, Curved in-plane electromechanical relay for low power logic applications, Journal of Micromechanics and Microengineering 23 (2) (2013) 025024.

URL http://stacks . iop.org/0960-1317/23/i=2/a=025024

[11] M. E. Schmidt, C. Xu, M. Cooke, H. Mizuta, H. M. H. Chong, Metal-free plasma-enhanced chemical vapor deposition of large area nanocrystalline graphene, Materials Research Express 1 (2) (2014) 025031.

URL http://stacks . iop.org/2053-1591/1/i=2/a=025031

[12] A. C. Ferrari, Raman spectroscopy of graphene and graphite: Disorder,electronphonon coupling, doping and nonadiabatic effects, Solid State Communications 143 (1) (2007) 47 - 57, exploring graphene. doi:https://doi.org/10.1016/j.ssc. 2007. 03.052

[13] K. Akarvardar, C. Eggimann, D. Tsamados, Y. Chauhan, G. Wan, A. Ionescu, R. Howe, H. P. Wong, Analytical modeling of the suspended-gate fet and design insights for low-power logic, IEEE Transactions on Electron Devices 55 (1) (2008) 4859. doi:10.1109/TED. 2007.911070 